\title{
Hemorrhagic Papular Eruption on the Dorsal Hands
}

Margaret E. Brown, MD; Frank Jing, BS; Huma A. Siddiqui, MD; Jennifer Krejci-Manwaring, MD
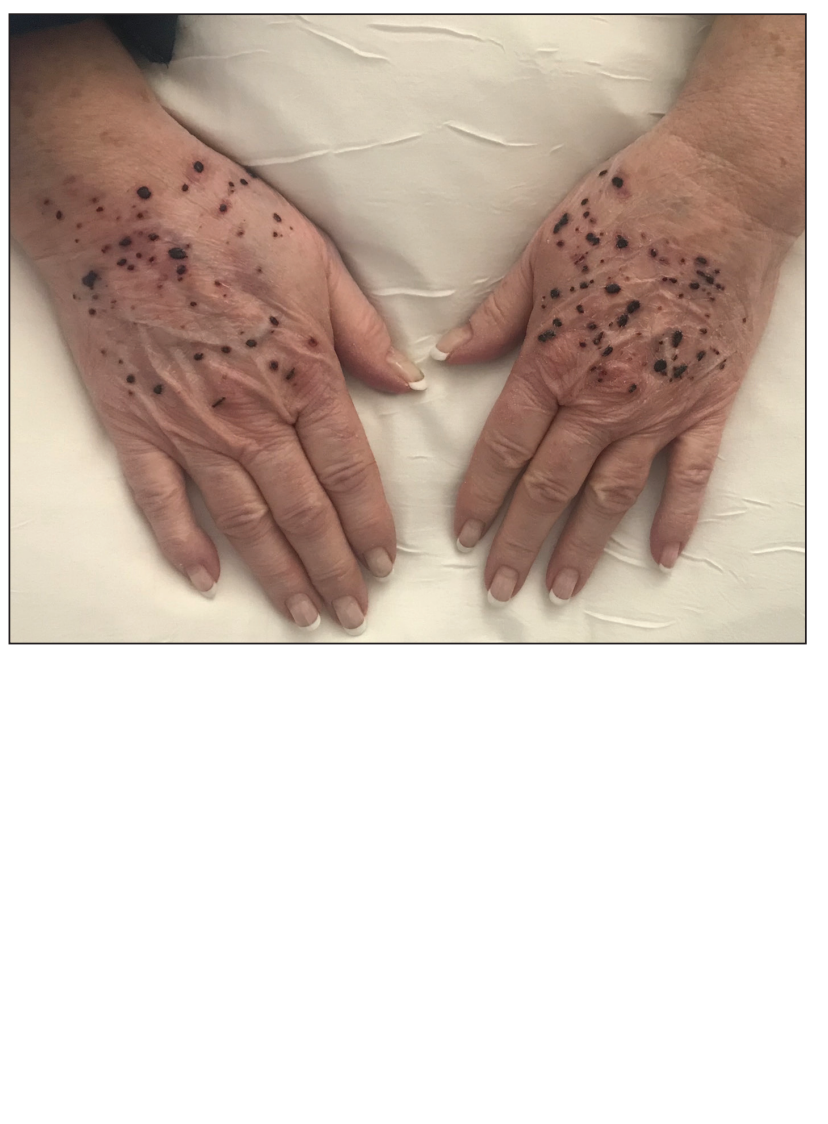

A 66-year-old woman with a history of granulomatous lung disease managed with methotrexate and prednisone, diabetes mellitus, hypertension, and Grave disease was admitted to the hospital for hypoxic respiratory failure. At admission, treatment was empirically initiated for pneumonia with intravenous ceftriaxone and azithromycin. Given the concern of a pulmonary embolism, intravenous heparin also was initiated. Dermatology was consulted for multiple painless blood blisters that erupted on the hands within 24 hours of admission. Physical examination revealed numerous firm hemorrhagic papules on the dorsal hands. Laboratory workup revealed a slightly elevated white blood cell count $(11,800 / \mu \mathrm{L}$ [reference range, $4500-11,000 / \mu \mathrm{L}])$, a normal stable platelet count $(231,000 / \mu \mathrm{L}$ [reference range, 150,000$350,000 / \mu \mathrm{L}])$, and a normal international normalized ratio.

\section{WHAT'S YOUR DIAGNOSIS?}
a. bullous drug eruption
b. disseminated herpes zoster
c. heparin-induced bullous hemorrhagic dermatosis
d. heparin necrosis
e. leukocytoclastic vasculitis

Drs. Brown and Krejci-Manwaring and Mr. Jing are from the Division of Dermatology \& Cutaneous Surgery, UT Health San Antonio. Dr. KrejciManwaring also is from and Dr. Siddiqui is from South Texas Veterans Health Care System, San Antonio.

The authors report no conflict of interest.

Correspondence: Margaret E. Brown, MD, Division of Dermatology \& Cutaneous Surgery, UT Health San Antonio, 7979 Wurzbach Rd, MC\# 7876 , San Antonio, TX 78229 (MargeBrownMD@gmail.com).

doi:10.12788/cutis. 0116 


\section{THE DIAGNOSIS: \\ Heparin-Induced Bullous Hemorrhagic Dermatosis}

R esults of a punch biopsy of one of the hemorrhagic papules revealed a subcorneal hemorrhagic vesicle without underlying vasculitis, vasculopathy, inflammation, or viral changes (Figure). Tissue and blood cultures were sterile. Heparin and platelet factor 4 antibody testing was negative. The patient was diagnosed with heparininduced bullous hemorrhagic dermatosis (BHD). After chest imaging ruled out a pulmonary embolism, anticoagulation therapy was discontinued. Respiratory symptoms improved on antibiotics, and the skin lesions resolved completely within 2 weeks.

Bullous hemorrhagic dermatosis is an uncommon and underrecognized reaction to various anticoagulants. Bullous hemorrhagic dermatosis presents with painless, noninflammatory, hemorrhagic vesicles and bullae occurring at sites distant from anticoagulant administration. The condition was first characterized in 2006 by Perrinaud et al, ${ }^{1}$ who presented 3 cases in patients treated with heparin and low-molecular-weight heparin. Since then, there have been at least 90 cases reported in the international literature, with elderly men found to be the more affected demographic (male to female ratio, 1.9:1). ${ }^{2}$ Typically, BHD presents within 1 week of administration of an anticoagulant, but delayed onset has been reported. ${ }^{2}$ Bullous hemorrhagic dermatosis is most commonly observed with enoxaparin use but also has been described in association with unfractionated heparin, low-molecular-weight heparin products, and warfarin. ${ }^{2}$

The noninflammatory-appearing hemorrhagic papules and small plaques of BHD generally are seen on the extremities but can occur anywhere on the body including the oral mucosa. ${ }^{3}$ The differential diagnosis of BHD may include autoimmune vesiculobullous conditions, bullous drug eruptions, herpetic infection, supratherapeutic anticoagulation, porphyria cutanea tarda, amyloidosis, leukocytoclastic vasculitis, angioinvasive infections, and heparin necrosis. Diagnosis of BHD can be made clinically, but a biopsy is useful to exclude other conditions.

Histologically, BHD is characterized by the presence of intraepidermal hemorrhagic bullae without thrombotic, inflammatory, or vasculitic changes. Although heparinrelated skin lesions have been attributed to various mechanisms, including immune-mediated thrombocytopenia, type IV hypersensitivity reactions, type I allergic hypersensitivity reactions, pustulosis, and skin necrosis, the pathogenesis of BHD remains poorly understood. ${ }^{4}$ The condition has demonstrated koebnerization in some cases. ${ }^{5}$

In our patient, the absence of histologic inflammation, viral changes, vasculitis, and amyloid deposition helped rule out the other entities in the differential. The absence of heparin and platelet factor 4 antibodies helped exclude

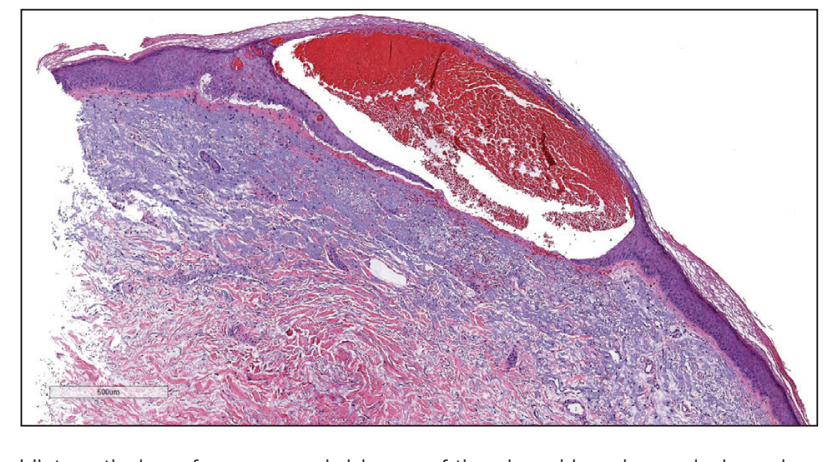

Histopathology from a punch biopsy of the dorsal hand revealed a subcorneal hemorrhagic vesicle without underlying vasculitis, vasculopathy, inflammation, or viral changes (H\&E, original magnification $\times 4$ ).

heparin necrosis. Direct immunofluorescence testing was not obtained in our patient but may be used to evaluate for an immunobullous etiology.

Management strategies for BHD are variable, and associated evidence is lacking. Treatment of BHD should be considered in the clinical context based on the necessity for anticoagulation and the severity of the eruption. Discontinuation of anticoagulation therapy, if possible, may prevent morbidity in some cases. ${ }^{6}$ If it is necessary to continue anticoagulation therapy, changing the drug or decreasing the dose are reasonable options. Skin lesions may resolve even if anticoagulation therapy is continued at the same dose. ${ }^{7,8}$ Concurrent supportive wound care is beneficial.

\section{REFERENCES}

1. Perrinaud A, Jacobi D, Machet MC, et al. Bullous hemorrhagic dermatosis occurring at sites distant from subcutaneous injections of heparin: three cases. J Am Acad Dermatol. 2006;54(suppl):S5-S7.

2. Russo A, Curtis S, Balbuena-Merle R, et al. Bullous hemorrhagic dermatosis is an under-recognized side effect of full dose low-molecular weight heparin: a case report and review of the literature [published online July 6, 2018]. Exp Hematol. 2018;7:15.

3. Harris HB, Kurth BJ, Lam TK, et al. Heparin-induced bullous hemorrhagic dermatosis confined to the oral mucosa. Cutis. 2019;103:365-366, 370.

4. Schindewolf M, Schwaner S, Wolter M, et al. Incidence and causes of heparin-induced skin lesions. CMAJ. 2009;181:477-481.

5. Gargallo V, Romero FT, Rodríguez-Peralto JL, et al. Heparin induced bullous hemorrhagic dermatosis at a site distant from the injection. a report of five cases. An Bras Dermatol. 2016;91:857-859.

6. Choudhry S, Fishman PM, Hernandez C. Heparin-induced bullous hemorrhagic dermatosis. Cutis. 2013;91:93-98.

7. Maldonado Cid P, Moreno Alonso de Celada R, Herranz Pinto P, et al. Bullous hemorrhagic dermatosis at sites distant from subcutaneous injections of heparin: a report of 5 cases. J Am Acad Dermatol. 2012;67:E220-E222.

8. Snow SC, Pearson DR, Fathi R, et al. Heparin-induced haemorrhagic bullous dermatosis. Clin Exp Dermatol. 2018;43:393-398. 